\title{
MODEL BASED DEFINITION: FINALLY, THE ENGINEERING DRAWING KILLER?
}

\author{
Nigel GARLAND, Russell WADE, Richard GLITHRO and Sarah PALMER-SMITH \\ Bournemouth University
}

\begin{abstract}
Engineering drawing has stood as the universal method of translating design intent since the first standard was formalised in 1927 as BS308. Further development of national and international standards has been informed by advances in CADCAM technology and the need for transfer of complex yet unambiguous definition between organisations. The emergence of model-based definition (MBD) has driven a new workflow where engineering drawing is no longer required. Instead, the dataset includes semantic, machine readable, tolerancing of surfaces and features for integration into manufacturing and metrology procedures. Despite the advantages of MBD, it has been largely ignored in UK higher education. However, MBD is the ideal method for teaching and learning geometrical tolerancing since it ignores the theoretically exact dimensions and housekeeping, concentrating on the functional limits. Further, it utilises the 3D workspace that students are increasingly familiar with.
\end{abstract}

Keywords: Model based definition, technical product specification, engineering drawing

\section{INTRODUCTION}

The end of the engineering drawing has been foretold many times since the first CAD systems began automating the generation of the technical product specification (TPS). However, despite the adoption of first 2D CAD and subsequently 3D CAD, the use of the 2D engineering drawing has persisted.

As CADCAM technology advanced it became routine to drive both manufacturing operations and metrology of complex parts directly from the geometrical model hence reduced reliance upon a full specification within the drawing. Instead, a hybrid method (combined 2D and 3D TPS) can be used where the drawing references the CAD model for theoretically exact dimensions (TEDs) derived from the referenced model with variance to nominal marked-up on the drawing itself. This solution can, however, lead to problems of precedent with the drawing considered the master document [1].

Alongside the integration of operations and move towards digital manufacturing there has been a continual development of engineering drawing practice (TPS) through international standards. Here, practice has developed over time through geometrical dimension and tolerancing (GD\&T) methodologies evolving into the ISO geometrical product specification (GPS) [2] and ASME Y14.5 standard [3]. Despite GD\&T being incorporated into British standards since 1953 [4] it has continued to be largely ignored within UK higher education. The lack of effective teaching in this area leaves many graduates unprepared for employment and at a distinct disadvantage compared to their international counterparts.

\subsection{Model Based Definition}

More recently, the 3D CAD environment has provided the backdrop for the application of specification directly to the model using model-based definition (MBD) or product manufacturing information (PMI) rather than within an engineering drawing. These methods lead to significant advantages in the transfer of requirement between organisations and processes such as translation from model to specification, manufacture and metrology per digital manufacturing workflow concepts such as Industry 4.0 [5]. For product lifecycle management (PLM) the model is essentially a digital twin [6] where the key feature is the semantic, machine readable, nature of the dataset; the annotation being a visual query of the dataset. Furthermore, the use of the tools restricts the source of data to a single point and therefore overcomes the problem of precedence, since there is no drawing, with the full specification part of the model file. A study within the Canadian aerospace sector found the majority of benefits from MBD will be found 
within the manufacturing and inspection [7], while document suitability for PLM has been evaluated in depth with IGES, STEP and 3D-pdf variants compared [8].

The emergence of GPS as the dominant method for specification lends itself ideal for conveying through PMI/MBD methodologies since the model itself is the TEDs and therefore no dimensioning is required; instead, it is the tolerances of size, location and surface variance (from the model) being controlled through geometrical tolerances. However, the widespread use of these methods is currently limited by interoperability, user knowledge and standards compliance [7] with very little effective learning material available to UK industry or higher education. It should be noted that MBD does not preclude the use of paper off-prints or conventional 2D TPS style layup since the model file holds precedence and output generated from it can be regarded in the same way as a photocopied drawing. Furthermore, MBD also holds significant advantage when applied to the assembly environment since the reader can orientate and hide components at will to enable a clear understanding of function, process and procedure.

\section{LEARNING STRATEGIES}

For this work, a combination of primary and secondary research methods were used. A review of the literature and existing learning materials was supplemented by primary research findings from our previous work on engineering drawing practice in education [9]. The outcomes of that work were also used to develop the strategy of the new work.

\subsection{Overview of TPS Literature}

The ISO-GPS method is well established and thoroughly documented but not well collated; instead they are controlled top down from ISO 8015 [2]. On the other-hand, the BS8888 [1] standard encompasses 137 British and international standards to provide a single, detailed, point of reference for the method described. For 3D annotation, such as MBD, the existing standards are further supplemented by ISO 16792 [10] and ASME Y14.41 [11] which provide a comprehensive set of rules for the practical application of GPS through MBD and describe the dataset as semantic. Alongside national and international standards, enterprise also embraces the hybrid and model based approach through supplier codes such as that used by Boeing [12]; here the hybrid method is described as a "reduced content drawing" with the supplier expected to extract addition information from the 3D model while suppliers receiving MBD datasets require a capability assessment by a Boeing quality rep. Although these standards provide a set of rules they are not designed to provide guidance on practical application or methodologies suitable as a learning tool. However, the BSI does publish a guide to engineering practice for higher education [13] but this is also rules based with emphasis upon "housekeeping" rather than specification. The BSI also publishes a document outlining objectives and learning outcomes for training [14] but is, again, rules based and essentially a set of lists rather than an effective workflow. Learning material in the form of published manuals and guidebooks also suffer from the legacy of 2D drawing methods and housekeeping and presentation [15] or rules based description [16, 17] rather than providing a methodology for establishing specification. MBD methods have traditionally been hampered by interoperability with data transfer being limited to CAD vendor specific file types. However, this expanded with the advent of Siemens JT format and the Adobe 3D pdf format which allowed data sharing without the original CAD modelling package. Data exchange has been greatly improved with development of the vendor neutral STEP AP242 [18] format which allows the communication of the full semantic MBD specification within a STEP solid model, although conversion between STEP242 and proprietary data types has been found to vary [19]. A study of the global aerospace sector identified the majority of benefits from MBD will be found within the manufacturing and inspection [7].

\subsubsection{Industrial Expectation}

Previous work [9] surveyed a panel of industrial experts using a structured questionnaire to identify core knowledge and expected learning outcomes. This found graduates are expected to have good knowledge and understanding of the underlying GPS methods such as basic GD\&T alongside the traditional "housekeeping" skills. Most also responded that students should have fair or good understanding of PMI/MBD. However, the panel reported actual knowledge and understanding amongst new graduates to be well below that expected. 


\subsection{Traditional 2D Drawing Learning Method}

Teaching of TPS has typically followed the traditional texts and methods derived from manual drawing methods. Essentially students typically learn the "housekeeping" of how to layout a drawing, use correct line types and weights, orthographic projection, chain and parallel dimension styles, borders, title blocks, and so on; essentially they were learning the presentational skills of draughting rather developing knowledge of how to convey design intent. Previous work [9] outlined these problems and provided a strategy and methodology for implementation of a four step procedure to provide clear design intent:

1. Operational (how does the assembly/system operate and what are the functional limits)

2. Functionality (form and size control of functional surfaces)

3. Kinematics (orientation and location between functional surface)

4. Bulk material (control of non-functional load carrying surfaces).

For MBD, much of the "housekeeping" is irrelevant since there are no orthographic views, no dimensions (other than size) and no border defining the paper space. However, the basic three steps outlined above are still required and are the essential part of defining the specification.

\subsection{Standards Compliance}

In developing new teaching material, it was deemed essential to mirror international standards and, at the same time, ensure learned concepts did not conflict between the three methods for TPS (2D drawing only, combined 2D and 3D, 3D only). Although there are differences between ASME Y14.5 and ISO GPS the underlying concepts are similar while, in the 3D MBD environment, ISO 16792 is actually an adaption of ASME Y14.41 rather than a stand-alone standard in its own right but also supplementary to ISO 1101 [20] which encompasses geometrical tolerancing.

ISO 8015 provides the fundamental rules for ISO GPS and offers some key guidance for understanding and developing a specification:

- Fundamental assumptions for reading the TPS; functional limits are based upon exhaustive investigation and are known with no uncertainty; it is assumed that the tolerance limits are identical to the functional.

- Independency principle; hence modifiers such as CZ, MMC, LMC and the envelope requirement.

- Duality principle; the specification shows what the designer wants (functional limits), not how to make it, or measure it.

For CAD software the integration of MBD is now common place with typical packages such as Dassault Systemes SolidWorks, Autodesk Inventor, PTC Creo and Siemens NX, amongst others, including such toolsets. However, the level of standards compliance and usability varies between applications; Creo and NX have extensive toolsets and high level of compliance while SolidWorks and Inventor less so.

\subsection{Generalised Workflow}

For successful application of GPS through MBD the functional limits should first be ascertained:

Standard parts and fittings should be identified and the manufacturer's, or standard's, data consulted to identify component limits.

Manufacturing process capability should be identified to ensure tolerances are reasonably achievable.

Designed parts should be allocated tolerances within the capability.

Tolerance stack analysis should be conducted to reference the overall functional limits.

The modelling process can follow or be in parallel to the analysis above but the application of the GPS specification to the parts using MBD should follow the modelling procedure and relate the analysis conducted and documented.

MBD application should follow the four-step procedure outlined earlier:

1. Understand the operation of system/assembly and the functional limits.

2. Functional surfaces should be identified, sized and toleranced.

3. Functional surfaces should be used as datums for controlling orientation, location and form of subsequent surfaces.

4. Non-functional surfaces should be controlled from the functional datum system.

For 3D MBD workflow there is (generally) no requirement for dimensions (other than size) as the parts are either: manufactured and measured directly from the model or, the dimensions can be derived through interrogation of the model. 


\section{PRACTICAL IMPLEMENTATION}

A new unit (Engineering Design Tools) was developed to instil knowledge and understanding of MBD to level 5 design engineering students. The students had limited understanding of GPS and TPS from level 4 but no knowledge of MBD. However, the same four step procedure developed for level 4 was applied within the MBD environment: understand system functionality; identify and classify functional surfaces; establish kinematic relationships of functional surfaces; control of remaining surfaces.

\subsection{Toolset Selection}

For this unit there was a trade-off between standards compliance, software familiarity, and software learning curve. Students were already experienced in the use of SolidWorks and therefore would be familiar with the interface and much of the toolset. Previous student experience with NX had found the complexity of the MBD tools overwhelming and therefore unsuitable for the timeframe available. Creo, while appearing to be the most suitable for the unit was not available at our institution. Inventor was discounted as, although the toolsets were similar to SolidWorks, students had less exposure to the interface; it was, however, deployed for design and specification of gears and shafts through its design accelerator in place of MITCalc [21] which had been used previously.

\subsection{Task}

Students were tasked to design a two-stage gearbox, and each provided with a unique combination of power rating, input speed and ratio. To accomplish this, students began by analysing the problem and identifying the functional limits through a basic workflow (Figure 1).
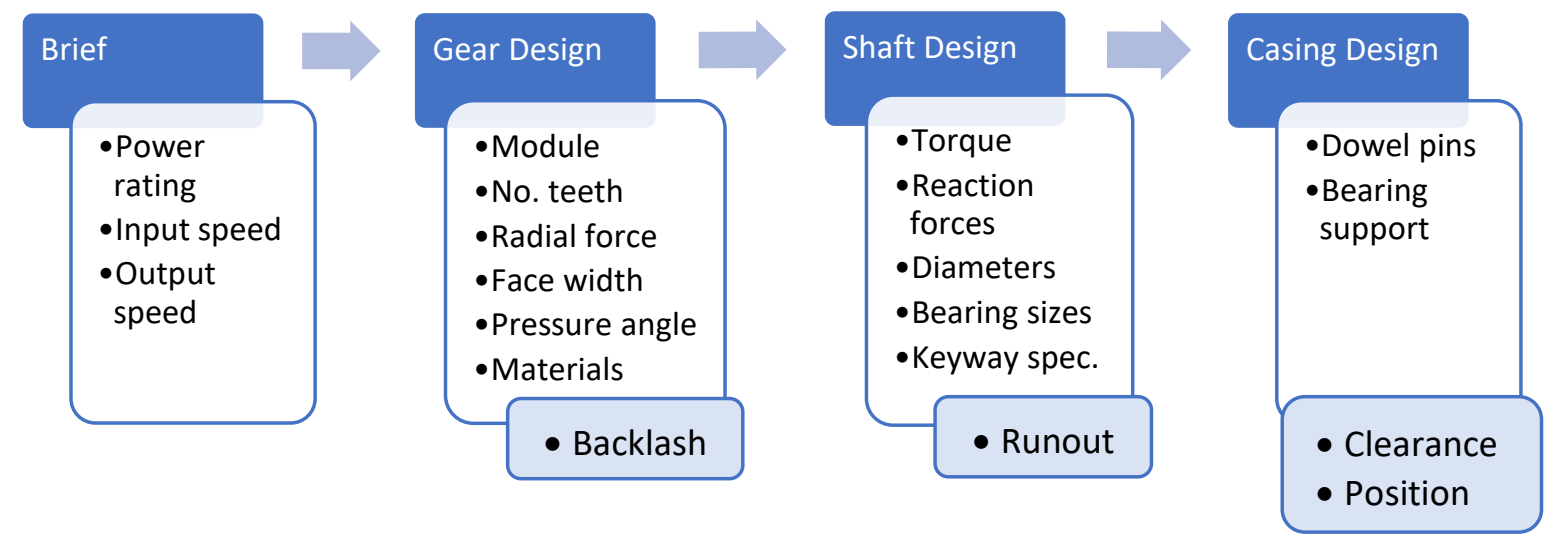

Figure 1 Specification and analysis workflow deriving functional limit and tolerances

After calculating suitable ratios for each stage, students used the Autodesk Inventor design accelerator package to optimise the design of the gearsets to inform load cases hence shaft, bearing and keyway specifications. With these specifications, students were able calculate the backlash required and therefore the overall functional limits of the assembly; these limits were then used to inform initial tolerances of component parts through collation of data in Excel and sketches. To develop understanding of the underlying concepts, teaching throughout the unit was whiteboard based before supplementing with YouTube video tutorials that were created on demand to address issues identified by the wider group.

Students developed a sound understanding of gearbox functionality through the design phase and practical support tutorials before they were introduced to MBD functionality and various layup methods of features of size, fits, and geometrical tolerances. Where appropriate, they applied tolerances recommended by mating-part manufacturers or standards; fits between shafts, bearings and housings were identified through SKF literature [22], while that for keyways was drawn from BS4235 [23]. Students followed the four-step procedure above and therefore identified and applied fits between components before orientation and location tolerances within components. As they worked through the components and interfaces they collated the tolerances for basic stack up analysis comprising shaft runout, bearing accuracy, clearance fits and position tolerances. These techniques were used to evaluate the projected alignment of the gears, hence control of backlash. Again, by focusing upon the functionality of the whole system, students developed understanding of the impact of tolerance selection. 
Alongside the basic control of gear alignment, they were introduced to pinned interfaces for the gearbox housing. Here the use of dowel pins was demonstrated with the use of the mating face and two dowels representing the basic datum system of plane-line-point.

\section{OUTPUT}

Students were expected to produce a full set of robust models and working assembly. Each designed part was fully annotated using MBD to control all surfaces; functional surfaces controlled discretely (Figure 2) while non-functional surfaces such as the casing body could be controlled using a surface profile with all-over modifier. They also produced examples of typical downstream output in the form of STEP 242 files. In addition, students were required to demonstrated understanding of the work done through a written report detailing the decision-making process used in the application of GPS through MBD.

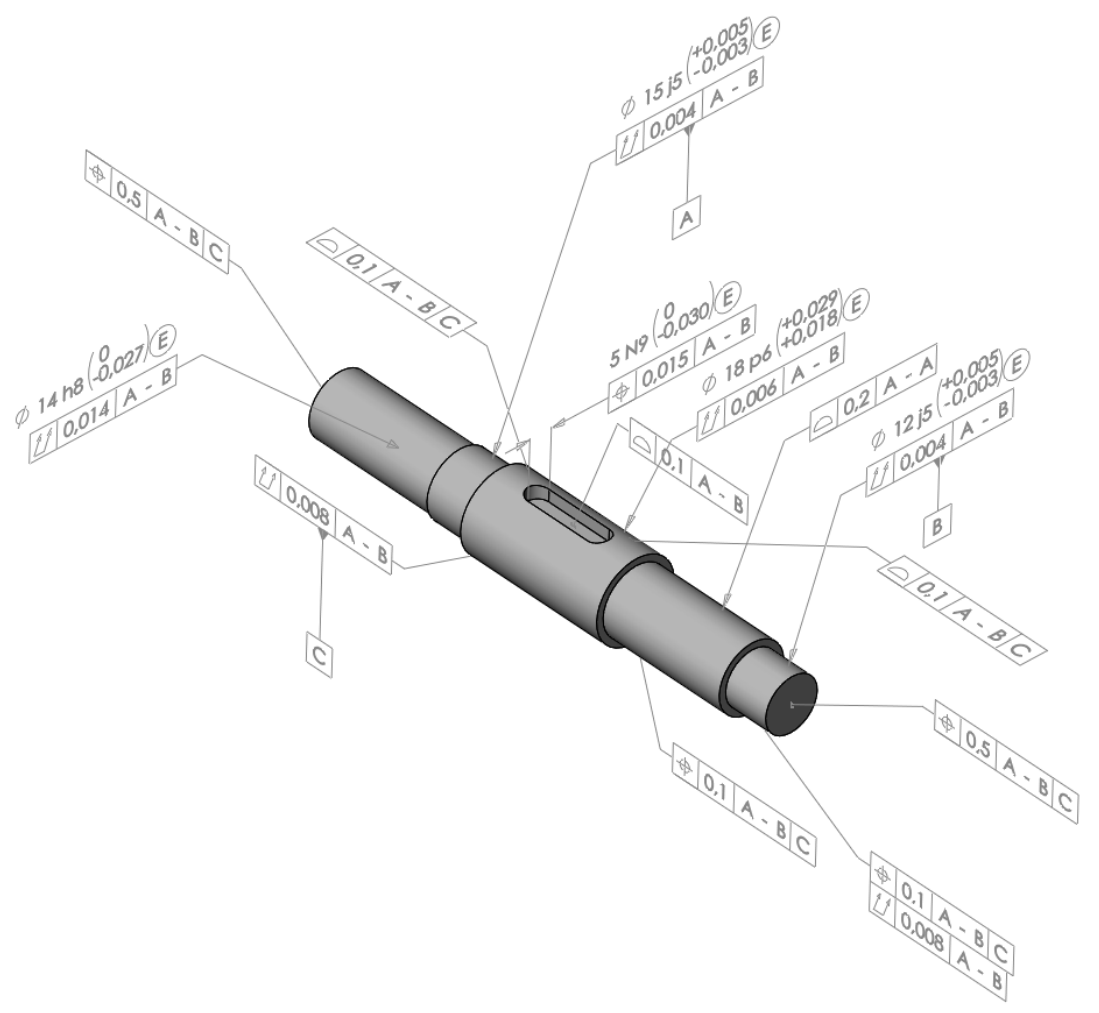

Figure 2 MBD applied to driveshaft; green surfaces indicate tolerance features are fully constrained

\subsection{Findings}

Students made fewer basic errors in annotation; this is not surprising since there is a single, orientable, view for annotation and students can visualise the model more readily than in 2D. Moreover, since the transition to 3D CAD modelling, students no longer develop geometry in 2D space and therefore 2D TPS could be viewed counter-intuitive for learning GPS.

Overall, students on this unit were found to develop a basic working knowledge of MBD and, more importantly, GPS; this knowledge is applicable whether using 2D or 3D TPS. Further, the clear focus of MBD upon the functional features guides the student thought process to follow a methodology that is equally valid whether using conventional 2D TPS or 3D TPS through MBD.

3D TPS through MBD provides a clear advantage over 2D TPS. By allowing annotation and allocation of tolerances during geometrical construction, the thought processes driving geometry and the control of fit and variance are linked; essentially, the functional limits and indication are tied.

Is MBD the engineering drawing killer? 


\section{REFERENCES}

[1] BSI, BS8888:2017 Technical product documentation and specification. 2017, British Standards Institution.

[2] ISO, Geometrical product specifications (GPS) - Fundamentals - Concepts, principles and rules (ISO 8015:2011). 2011, International Organization for Standards.

[3] ASME, Dimensioning and Tolerancing (ASME Y14.5-2009). 2009, The American Society of Mechanical Engineers.

[4] BSI, Engineering Drawing Practice, British Standard BS 308: 1953. 1953: British Standards Institution.

[5] Zhigang Fang F., Li Z., Arokiam A. and Gorman T. Closed Loop PMI Driven Dimensional Quality Lifecycle Management Approach for Smart Manufacturing System. Procedia CIRP, 2016. 56: p. 614 - 619.

[6] Schleich B., Wärmefjord K., Söderberg R. and Wartzack S. Geometrical Variations Management 4.0: towards next Generation Geometry Assurance. Procedia CIRP, 2018. 75: p. 3 - 10.

[7] Quintana V., et al., Will Model-based Definition replace engineering drawings throughout the product lifecycle? A global perspective from aerospace industry. Computers in Industry, 2010. 61(5): p. 497-508.

[8] Pfouga A. and Stjepandić J. Leveraging 3D geometric knowledge in the product lifecycle based on industrial standards. Journal of Computational Design and Engineering, 2018. 5(1): p. 54 67.

[9] Garland N., Glithro R. and Wade R. The Challenges Facing Education in Engineering Drawing Practice, in 19th International Conference on Engineering and Product Design Education., A. Berg, et al., Editors. 2017, The Design Society, Institution of Engineering Designers.

[10] ISO, Technical product documentation — Digital product definition data practices (ISO 16792:2015). 2015, International Organization for Standards.

[11] ASME, Digital Product Definition Data Practices (ASME Y14.41-2012). 2012, The American Society of Mechanical Engineers.

[12] Boeing, Quality Assurance Standard for Digital Product Definition at Boeing Suppliers (d651991 Rev L). 2016, Boeing Management Company.

[13] BSI, Phelps N. and Simmons C.H. PP8888-2: 2007 Engineering Drawing Practice. A Guide for Further and Higher Education to BS 8888: 2006, Technical Product Specification (TPS). 2007: British Standards Institution.

[14] BSI, PD 68888:2011 Objectives and learning outcomes for BS 8888 training. 2011, British Standards Institution.

[15] Simmons, C.H. and D.E. Maguire, Manual of Engineering Drawing: Technical Product Specification and Documentation to British and International Standards. 2012: Elsevier Science.

[16] Nielsen H.S. The ISO Geometrical Product Specifications Handbook: Find Your Way in GPS. Danish Standards.

[17] Charpentier F. and AFNor, Handbook for the Geometrical Specification of Products: The ISOGPS Standards. 2013: Afnor.

[18] ISO, Industrial automation systems and integration -- Product data representation and exchange -Part 242: Application protocol: Managed model-based 3D engineering (ISO 10303-242:2015). 2015, International Organization for Standards.

[19] Bijnens J., Kellens, K. and Cheshire D. Accuracy of geometry data exchange using STEP AP242. Procedia CIRP, 2018. 78: p. 219 - 224.

[20] ISO, Geometrical product specifications (GPS) - Geometrical tolerancing — Tolerances of form, orientation, location and run-out (ISO 1101:2017). 2017, International Organization for Standards.

[21] Petele M. MITCalc. 2018, MITCalc: Decin, Czech Republic.

[22] SKF. Bearing Selection Process. 2018; Available from: https://www.skf.com/uk/products/bearings-units-housings/principles/bearing-selectionprocess/index.html.

[23] BSI, Specification for Metric keys and keyways — Part 1: Parallel and taper keys (BS42351:1972). 1972, British Standards Institution. 